\title{
Read Range Limitation in IF-Based Far-Field RFID Using ASK Backscatter Modulation
}

\author{
Nicolas Pillin, Catherine Dehollain and Michel J. Declercq \\ Electronics Laboratory of the Swiss Federal Institute of Technology (EPFL), CH-1015 Lausanne, Switzerland \\ Email: \{nicolas.pillin, catherine.dehollain, michel.declercq\}@epfl.ch
}

\begin{abstract}
A model is proposed to describe the fundamental read range limitation due to the local oscillator phase noise in the reader, in IF-based, far-field RFID systems using amplitude-shift keying backscatter modulation. The relation between the system parameters (such as the data transfer rate) and the read range is discussed. The model is validated by measurements done on two different laboratory tag-reader systems.
\end{abstract}

\section{INTRODUCTION}

Passive, far-field RFID tags collect a fraction of the RF power sent by the reader to generate their own supply voltage. They transmit data back to the reader by modulating the waves that are reradiated by their antenna (backscatter modulation). On the one hand, the remote powering of a tag is limited to a specific powering range. It is the distance where the power available at the tag antenna terminals is just sufficient for a correct operation of the tag. On the other hand, the communication link is also limited to a specific read range. It corresponds to the distance where the backscattered power received by the reader is just sufficient for a correct detection of the transmitted data.

Designing a competitive RFID system often requires to optimize the operating range. This asks to determine whether the powering or the read range fixes the maximum distance, and to understand how it can be further increased. To this end, it is mandatory to model both aspects of RFID systems. Remote powering has already been extensively addressed in the literature (e.g. [1], [2], [3]). Concerning the read range, the fact that the local oscillator (LO) phase noise in the reader is the main concern has already been emphasized [2], [3], [4]. A detailed theoretical analysis has been proposed in the case where phase-shift keying (PSK) backscatter modulation is used [3]. The present paper proposes a model that focuses on amplitude-shift keying (ASK). The model is validated by measurements done on two different laboratory RFID systems. This model is also compatible with semi-passive, IF-based RFID systems with tags using ASK backscatter modulation. In this case, the operating range is equal to the read range, as there is no need for remote powering.

This paper begins with a description of the general communication principle in passive and semi-passive far-field RFID, in Section II. Section III discusses a model to estimate the read range. The model is validated by measurements done on two laboratory tag-reader systems in Section IV.

\section{COMMUNiCATION PRINCIPLE IN FAR-FIELD RFID}

A simplified view of a typical far-field tag-to-reader communication link through backscatter modulation is shown in Figure 1. This view focuses on the key parts of the system concerning the communication link, and the majority of the tag's sub-systems are omitted, including for instance the demodulator and the rectifier (if the tag is passive.) The Tx path of the reader contains a LO that generates a carrier signal at frequency $f_{R F}$. The LO feeds a power splitter cascaded with a power amplifier that drives the Tx antenna. The total gain from the LO's output to the Tx antennas terminals is named $G_{T x}$. This gain accounts for the loss due to the power splitter $C_{s p l}$ and to the gain of the power amplifier $G_{a m p}$. In the tag, a subcarrier is generated by an intermediate frequency (IF) oscillator with frequency $f_{I F}$. A line code is applied to the IF signal according to the baseband data to be transmitted. The resulting signal controls a switch that modifies the antenna load. In Fig. 1, the switch is either connected to an open-circuit or a short-circuit. In practice any combination of complex impedances can be used. The switch creates a modulated backscattered signal that contains the baseband data, as the tag antenna reradiates a signal whose amplitude and phase depends on the antenna load. The Rx path of the reader consists of a Rx antenna, a LNA and a mixer that performs a first downconversion of the useful signal. It is then sent to subsequent signal processing circuits that perform the final downconversion to baseband (not shown in Fig. 1). The overall architecture forms a low-IF receiver.

\section{READ RANGE LIMITATION DUE TO TX/RX COUPLING}

The read range of the RFID system depicted in Figure 1 depends on the sensitivity of the reader, which is related to the noise and interferers levels at the $\mathrm{Rx}$ antenna terminals (point $A$ ). The larger the noise and the effects of the interferers, the larger the required received signal power to get a satisfying SNR, and thus the smaller the read range. The interferers may degrade the sensitivity in various ways, and they typically generate parasitic signals and noise in the useful frequency band [1], [5], [6]. Supposing that this issue is properly addressed, a certain noise level still remains at point $A$. In the ideal case, this noise floor is due to thermal noise only. In practice, the isolation between the $\mathrm{Tx}$ and $\mathrm{Rx}$ antennas isn't perfect and a parasitic coupling $C_{T x / R x}$ exists. The LO phase noise propagates to point $A$ and raises the noise floor to a much higher level, causing a fundamental limitation in 


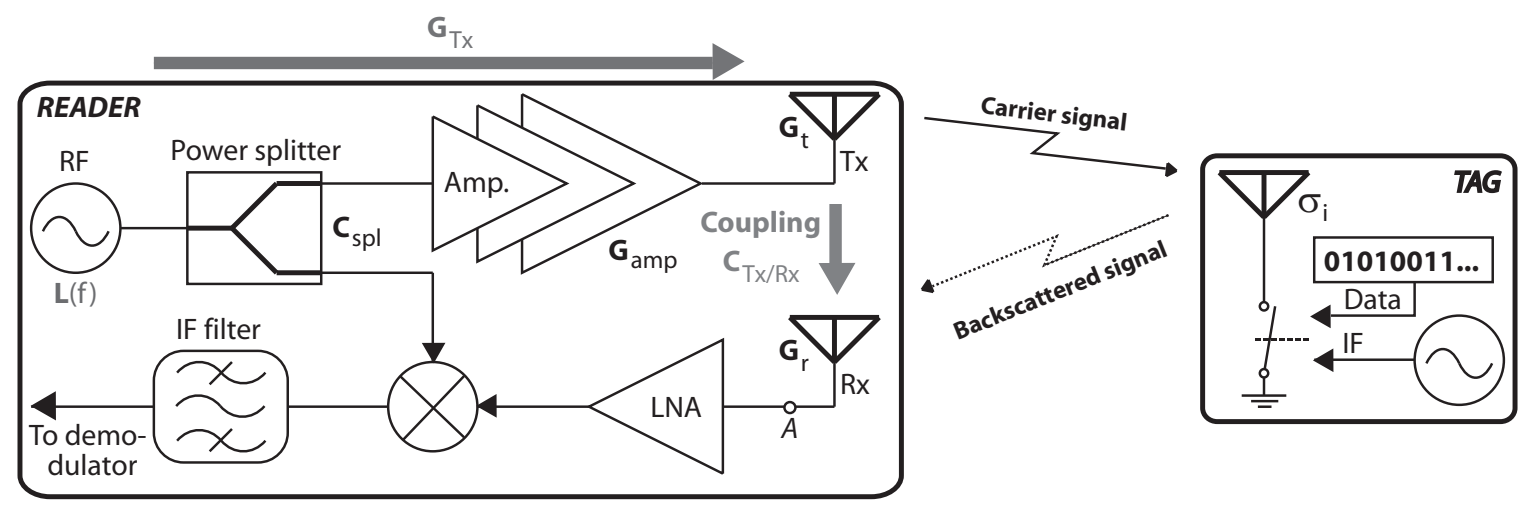

Fig. 1. Simplified view of a typical far-field tag-to-reader communication link through backscatter modulation.

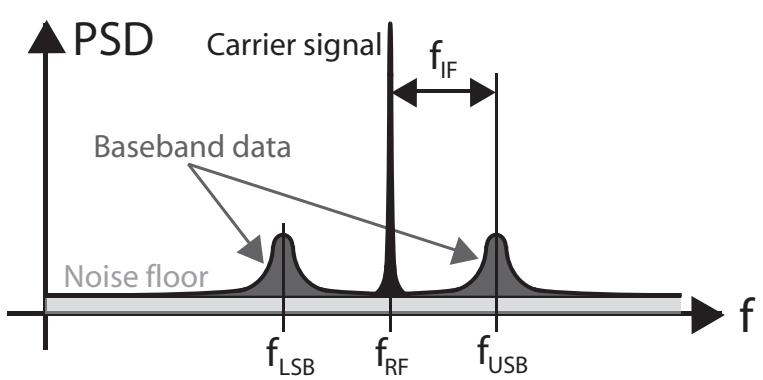

Fig. 2. PSD of the backscattered signal received at point $A$ in Fig. 1

terms of reader sensitivity [2], [3], [4]. The reader architecture described in this paper uses two antennas. The use of a single antenna combined to a circulator or a directional coupler is also possible [1], [7]. The below analysis still applies; in this case, $C_{T x / R x}$ accounts for the parasitic Tx-to-Rx coupling in the circulator or the directional coupler.

Figure 2 shows a simplified view of the received PSD at point $A$. It consists of the sum of the received backscattered signal and of the emitted carrier signal received by $\mathrm{Tx} / \mathrm{Rx}$ coupling, which is several order of magnitude larger. The two sidebands centered around frequencies $f_{L S B}=f_{R F}-f_{I F}$ (lower sideband or LSB), and $f_{U S B}=f_{R F}+f_{I F}$ (upper sideband or USB) contain the baseband data. The noise floor is a function of the LO phase noise that reaches the Rx antenna terminals through $\mathrm{Tx} / \mathrm{Rx}$ coupling.

Let's call $L(f)$ the PSD of the LO signal, normalized to the LO output power $P_{L O} . L(f)$ accounts for the phase noise, plus any spurious or amplitude noise that may also be generated by the oscillator. All these parasitics are transmitted to point $A$ and raise the noise floor. The spectral occupation of the useful signal corresponds to a bandwidth $B$ around $f_{L S B}$ and $f_{U S B} . B$ is related to the data transfer rate $R$. For typical line coding techniques, the ratio $B / R$ is either equal to 2 or 4 [5]. Let's define $N(f, B)$, the total noise power in a bandwidth $B$ centered around $f$, normalized to $P_{L O}$ :

$$
N(f, B)=\int_{f-B / 2}^{f+B / 2} L(f) d f
$$

The noise power is proportional to $N\left(f_{L S B}, B\right)$ for the LSB, and to $N\left(f_{U S B}, B\right)$ for the USB. The PSD of the LO signal is essentially symmetric around the carrier frequency $f_{R F}$ and these figures are almost equal. The emitted carrier power can be calculated as

$$
P_{e}=P_{L O} C_{s p l} G_{a m p} G_{t}=P_{L O} G_{T x} G_{t}
$$

where $G_{t}$ is the gain of the $\mathrm{Tx}$ antenna, and $G_{T x}=C_{s p l} G_{a m p}$. The power $P_{b}$ that is backscattered by the tag antenna depends on the module $\sigma$ of its radar cross-section (RCS). In this paper, we'll assume for simplicity there is no multipath effect [2], [7], [8]. Assuming that the $\mathrm{Tx}$, tag, and Rx antennas are perfectly aligned and that there are no polarization losses, we get

$$
P_{b}=\frac{P_{e}}{4 \pi d^{2}} \sigma
$$

where $d$ is the tag-reader distance. The power density of the backscattered signal at the reader side is given by

$$
S_{b}=\frac{P_{b}}{4 \pi d^{2}}=\frac{P_{e}}{(4 \pi)^{2} d^{4}} \sigma
$$

This relation determines the received power density for any radar cross-section $\sigma$. In our case, the switch in the tag varies the antenna load between two different impedances, which corresponds to two particular RCS's $\sigma_{1}$ and $\sigma_{2}$. The power density $S_{b}$ is related to the effective electric field strength $E_{b}$ in the following way [2]:

$$
S_{b}=\frac{E_{b}^{2}}{120 \pi}
$$

Combining (5) with (4) yields

$$
E_{b}=\frac{\sqrt{120 \pi P_{e}}}{4 \pi d^{2}} \sqrt{\sigma}=\epsilon \sqrt{\sigma}
$$

where $\epsilon=\sqrt{120 \pi P_{e}} /\left(4 \pi d^{2}\right)$. We define the received effective fields strength $E_{b 1}$ and $E_{b 2}$ for both modulation states, corresponding to the RCS's $\sigma_{1}$ and $\sigma_{2}$, respectively. We will assume for simplicity that the IF signal has a $50 \%$ duty cycle. We can rewrite $E_{b}$ as 


$$
E_{b}(t)=E_{b c}[1+m(t)]
$$

with

$$
\begin{gathered}
E_{b c}=\frac{E_{b 1}+E_{b 2}}{2}=\epsilon \frac{\sqrt{\sigma_{1}}+\sqrt{\sigma_{2}}}{2} \\
m(t)=\frac{E_{b 1}-E_{b 2}}{E_{b 1}+E_{b 2}} f(t)=\frac{\sqrt{\sigma_{1}}-\sqrt{\sigma_{2}}}{\sqrt{\sigma_{1}}+\sqrt{\sigma_{2}}} f(t)
\end{gathered}
$$

where $f(t)$ is an alternative signal that varies between +1 and -1 with a frequency $f_{I F}$ and a duty cycle of $50 \%$. $E_{b c}$ is the average effective field strength that reaches the Rx antenna. This field is multiplied by $m(t)$ to account for the modulation caused by the periodic load switching. The received power density can now be written as [5]:

$$
S_{b}=\frac{E_{b}^{2}}{120 \pi}=\frac{E_{b c}^{2}}{120 \pi}+\frac{E_{b c}^{2}}{120 \pi}\left\langle m(t)^{2}\right\rangle=S_{b c}+S_{b i}
$$

$S_{b c}$ conveys the discrete carrier power and $S_{b i}$ the sideband power that contains information. We have

$$
S_{b i}=\frac{P_{e}}{(4 \pi)^{2} d^{4}} \sigma_{i}
$$

with

$$
\sigma_{i}=\left(\frac{\sqrt{\sigma_{1}}-\sqrt{\sigma_{2}}}{2}\right)^{2}
$$

As the useful power density spreads into two main sidebands and also into subsequent sidebands created by the harmonics of the IF signal, a conversion loss factor $\alpha$ is defined that gives the fraction of $S_{b i}$ that actually goes into each main sideband. The line code applied to the IF signal according to the baseband data causes additional losses, which are also accounted for in $\alpha$. The received power in any sideband is obtained from (11):

$$
P_{S B}=\alpha S_{b i} \frac{\lambda^{2}}{4 \pi} G_{r}
$$

where $G_{r}$ is the receiving antenna gain, and $S B$ stands for $L S B$ or $U S B$. Combining the last equation with (2) and (11) yields

$$
P_{S B}=P_{L O} G_{T x} G_{t} G_{r} \alpha \sigma_{i} \frac{\lambda^{2}}{(4 \pi)^{3} d^{4}}
$$

The total noise power in the sidebands due to the $\mathrm{Tx} / \mathrm{Rx}$ coupling is obtained by using (1) and observing Fig. 1:

$$
N_{S B}=P_{L O} G_{T x} C_{T x / R x} N\left(f_{S B}, B\right)
$$

The SNR in any sideband is given by the ratio $P_{S B} / N_{S B}$. Combining (14) and (15), and defining $S N R_{\min }$ as the minimum required SNR, we obtain the corresponding read range $d_{\text {max }}$ :

$$
d_{\max }=\sqrt[4]{\frac{G_{t} G_{r} \alpha \sigma_{i}}{C_{T x / R x} N\left(f_{S B}, B\right) S N R_{\min }} \frac{\lambda^{2}}{(4 \pi)^{3}}}
$$

Given any maximum authorized BER at the baseband, the corresponding $S N R_{\min }$ at point $A$ can be calculated [5], and the read range $d_{\max }$ can be estimated according to the remaining parameters. It must be pointed out that the reader behaves as a radar (the same LO is used for Tx and Rx), and that range correlation occurs [6]. This effect must be taken into account when calculating $S N R_{\min }$ for a specific BER.

We see that the read range increases if the $G \lambda$ product of the Tx and Rx antennas improves. Note that the gain and the resonance frequency of an antenna aren't independent and that optimizing this product generally asks for larger antennas. $d_{\max }$ also increases with better LO phase noise performance and/or better $\mathrm{Tx} / \mathrm{Rx}$ isolation. The parameters $\sigma_{i}$ and $\alpha$ basically describe how efficiently the available power at tag side is converted into information. The range improves if $\sigma_{i}$, which is proportional to the differential RCS of the tag [7], becomes larger. The best situation is to switch between a shortcircuit and an open-circuit [2], [7], but no power is available for the tag in that case. Actually, there is a strict trade-off between the quality of the communication and the available power through remote powering [1]. As the line code has an impact on $\alpha$ and on the ratio $B / R$, particular care should be taken when choosing the coding technique in order to optimize the communication efficiency. Looking back to (1), we see that the lower the data rate $R$ (and thus the lower $B$ ), the lower the noise power, which converts in a larger $d_{\max }$. Finally, it is advantageous to work at high IF, as the LO phase noise is lower at larger offsets from the center frequency. However, this comes at the cost of a higher tag power consumption.

\section{Measurement Results}

In order to verify the validity of (16), measurements were achieved at $900 \mathrm{MHz}$ and $2.45 \mathrm{GHz}$ with two different laboratory tag-reader systems. All these measurements were done in the USB for a target SNR of $20 \mathrm{~dB}$. In the reader, the LO signal is created by a broadband signal generator that directly drives a Tx antenna. The data to be sent by the tags is generated by a pseudo-random number generator. The generator output sequence is connected to the first input of an AND gate, while the second input is connected to an IF oscillator. The output of the AND gate drives a RF switch in series with the antenna. A block diagram of both tags is shown in Figure 3. The signal at the receiving antenna terminals is amplified by a LNA before reaching a spectrum analyzer for the measurement of the SNR. The relevant system parameters are summarized in Table I. $N\left(f_{U S B}, B\right)$ was measured in the USB at $910 \mathrm{MHz}$ and $2.46 \mathrm{GHz}$ for all tested data rates. The measurement results are shown in Table II.

The IF signal is a square wave with a duty cycle of $50 \%$. It drives, through the AND gate, a switch that connects the antenna to a load $Z_{1}$ and $Z_{2}$ alternatively, which generates an ASK backscattered signal. In this situation, it can be shown that $40 \%$ of the power of the varying field is transferred in each main sideband. The bit sequence to be transferred controls the other input of the AND gate; the switch oscillates when the data line is HIGH, whereas it stops oscillating when the data 


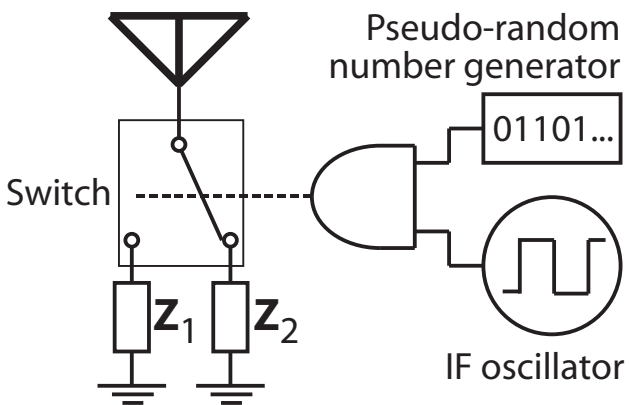

Fig. 3. Block diagram of the tags architecture used for the measurements.

\begin{tabular}{|l|c|c|c|}
\hline Name & Tag @ 900 MHz & Tag @ 2.45 GHz & Unit \\
\hline \hline$f_{R F}$ & 900 & 2450 & $\mathrm{MHz}$ \\
\hline$\lambda$ & 33.3 & 12.2 & $\mathrm{~cm}$ \\
\hline$f_{I F}$ & 10 & 10 & $\mathrm{MHz}$ \\
\hline$G_{t}$ & 2 & 7 & $\mathrm{dBi}$ \\
\hline$G_{r}$ & 2 & 7 & $\mathrm{dBi}$ \\
\hline$C_{T x / R x}$ & -23 & -27 & $\mathrm{~dB}$ \\
\hline$Z_{1}$ & 50 & 50 & $\Omega$ \\
\hline$Z_{2}$ & 0 & $\infty$ & $\Omega$ \\
\hline$\sigma_{i}$ & 64.4 & 17.4 & $\mathrm{~cm}^{2}$ \\
\hline$\alpha$ & -7 & -7 & $\mathrm{~dB}$ \\
\hline
\end{tabular}

TABLE I

SYSTEM PARAMETERS OF THE LABORATORY TAG-READER SYSTEMS

line is LOW. This technique, known as on-off keying (OOK,) has a $B / R$ ratio of $2[5]$ and $50 \%$ of the backscattered power is lost on average, assuming the same probability for a " 0 " or a " 1 ". The resulting value for $\alpha$ is $-7 \mathrm{~dB}$. The value of $\sigma_{i}$ was obtained by measuring the RCS of the tag antenna for both modulation states and by using (12).

Using the parameters of Tables I and II in (16), with $S N R_{\min }=20 \mathrm{~dB}$, the theoretical curves were plotted in Figure 4. Also shown are the measurements results. We see that the model is in good agreement with the measurements. $d_{\max }$ drops at higher data rates, as predicted by (16). This is due to the larger total noise power at the Rx antenna terminals at larger useful bandwidths, as can be seen from Table II. It can also be seen that the system at $900 \mathrm{MHz}$ reaches larger distances than the system at $2.45 \mathrm{GHz}$. This is mainly related to the lower propagation losses at $900 \mathrm{MHz}\left(\lambda^{2}\right.$ factor in (16)). The small errors between the theoretical curves and the measurements are mainly due to the none perfectly anechoic

\begin{tabular}{|c|c|c|c|}
\hline$R[\mathrm{kbps}]$ & $B[\mathrm{kHz}]$ & $N @ 910 \mathrm{MHz}[\mathrm{dBc}]$ & $N @ 2.46 \mathrm{GHz}[\mathrm{dBc}]$ \\
\hline \hline 100 & 200 & -80 & -72 \\
200 & 400 & -77 & -69 \\
500 & 1000 & -72 & -64 \\
1000 & 2000 & -68 & -61 \\
2000 & 4000 & -64 & -57 \\
5000 & 10000 & -60 & -52 \\
\hline
\end{tabular}

TABLE II

$N(f, B)$ FOR THE VARIOUS DATA RATES TESTED DURING THE MEASUREMENTS, FOR $f=910 \mathrm{MHz}$ AND $f=2.46 \mathrm{GHz}$.

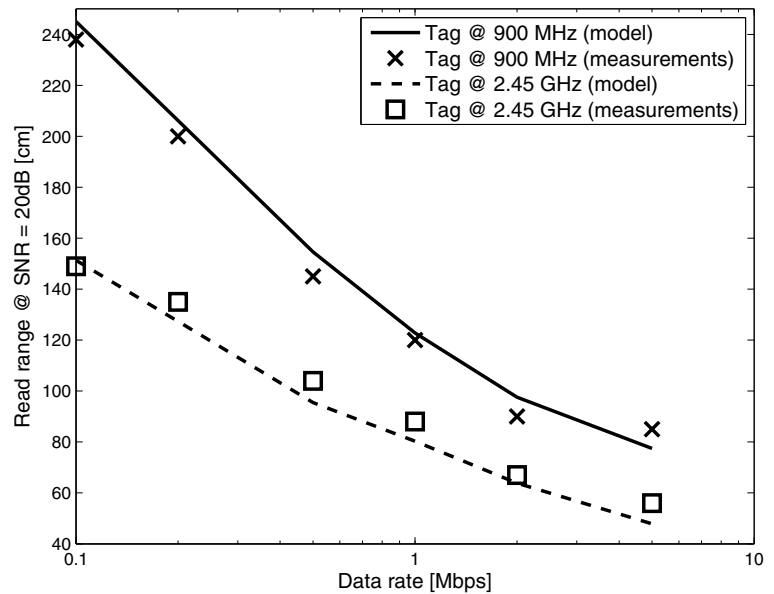

Fig. 4. Read range versus data rate with a target SNR of $20 \mathrm{~dB}$, for the two different tag-reader systems described in Table I.

environment (dispersion of the measurement points) and to the limited accuracy of the set-up used for RCS measurements (static error.)

\section{CONCLUSION}

The fundamental limitation in terms of read range due to the LO phase noise in the reader was analyzed for passive and semi-passive far-field RFID systems that use an IF with ASK backscatter modulation. The read range $d_{\max }$ can be estimated with good accuracy for a variety of data rates and minimum desired SNR at the Rx antenna terminals. The effect of the various system parameters on $d_{\max }$ was discussed. The proposed model was validated by measurements achieved on two different tag-reader systems.

\section{ACKNOWLEDGMENT}

This work was supported in part by the European Commission under FP6 Contract IST-034690 within the framework of the European Integrated Project MINAmI.

\section{REFERENCES}

[1] J.-P. Curty, M. Declercq, C. Dehollain, and N. Joehl, Design and optimization of passive UHF RFID systems, 1st ed. Springer, 2007.

[2] K. Finkenzeller, RFID handbook, 2nd ed. John Wiley \& Sons, 2003.

[3] G. D. Vita and G. Iannaccone, "Design criteria for the RF section of UHF and microwave passive RFID transponders," in IEEE Trans. on Microwave Theory and Techniques, 2005, pp. 2978-2990.

[4] D. M. Dobkin. (2005, April) The RF in RFID: physical layer operation of passive UHF tags and readers. Enigmatics. [Online]. Available: http://www.enigmaticconsulting.com/Communications_articles/RFID/RF_in_RFID_index.html

[5] L. W. Couch, Digital and analog communication systems, 6th ed. Prentice-Hall, 2000.

[6] J. H. Bae, J.-C. Kim, B.-W. Jeon, J.-W. Jung, J.-S. Park, B.-J. Jang, H.-R. Oh, Y.-J. Moon, and Y.-R. Seong, "Analysis of phase noise requirements on local oscillator for RFID system considering range correlation," in 37th European Microwave Conf., 2007, pp. 1664-1667.

[7] P.-V. Nikitin and K. V. S. Rao, "Antennas and propagation in UHF RFID systems," in IEEE Int. Conf. on RFID, 2008, pp. 277-288.

[8] S. R. Banerjee, R. Jesme, and R. A. Sainati, "Performance analysis of short range UHF propagation as applicable to passive RFID," in IEEE Int. Conf. on RFID, 2007, pp. 30-36. 